\title{
A RELAÇÃO V/VE DA RADIAÇÃO SOLAR SOB O DOSSEL DE UMA ÁREA DE MATA ATLÂNTICA
}

\author{
José Eduardo Zaia' \\ Massanori Takaki
}

Recebido em 06/08/1998. Aceito em 17/03/1999

\begin{abstract}
RESUMO - (A relação V/VE da radiação solar sob o dossel de uma área de Mata Atlântica). A distribuição espectral da radiação foi obtida nos meses de agosto de 1994, junho e agosto de 1995 numa área de Mata Atlântica situada no Parque Estadual Intervales, Base do Saibadela $\left(24^{\circ} 14^{\circ} \mathrm{S}\right.$ e $\left.48^{\circ} 04^{\circ} \mathrm{W}\right)$. município de Sete Barras, SP. As leituras de varredura do espectro foram realizadas com espectroradiômetro LI-1800. em 22 pontos sob o dossel da floresta e três pontos numa área aberta, em condições de céu aberto ( $\mathrm{sol}$ ) e céu encoberto (nublado). Para a análise foram utilizadas as variáveis PPFD e a relação V/VE (655-665/725-735nm). A qualidade da luz fora do dossel não foi influenciada pela radiação difusa. Sob o dossel, foi observado aumento significativo $(\mathrm{P}<0.05)$ da relação V/VE $(0,54 \pm 0,08)$ em condição de céu nublado comparado com a condição de céu aberto $(0.27 \pm 0.07)$. Esta grande alteração na qualidade de luz. devido aos longos períodos de chuva e céu encoberto que ocorrem com muita frequiência na região, pode ser de grande relevância para os processos fotomorfogênicos das espécies vegetais que ocorrem neste ambiente.
\end{abstract}

Palavras-chave - radiação solar, Mata Atlântica, fitocromo, V/VE

\begin{abstract}
The R/FR ratio of solar radiation under the canopy in an area of the Atlantic Forest). The spectral distribution of solar radiation was obtained during August 1994 and June and August 1995, in an area of the Atlantic Forest at "Parque Estadual Intervales, Base do Saibadela" (24"14'S and $\left.48^{\circ} 04^{\prime} \mathrm{W}\right)$, Sete Barras, SP. The light spectra were obtained with the aid of a LI-1800 spectroradiometer in 22 spots under canopies, and three spots in an open area under conditions of overcast and sunny day. The PPFD and R/FR ratio (655-665/725-735nm) were used for radiation analysis. The quality of light was not influenced by the clouds but under the canopy there were significant differences $(P<0.05)$ in the $R / F R$ ratio $(0.54 \pm 0.08)$ in overcast and sunny day conditions $(0.27 \pm 0.07)$. This alteration in the quality of light due to the long periods of rainy days in the region can be of great significance for the photomorphogenic processes of plants that occur in this environment.
\end{abstract}

Key words - solar radiation, Atlantic Forest, phytochrome, R/FR

\section{Introdução}

A radiação que atravessa o dossel de uma floresta sofre alterações na sua composição espectral, sendo caracterizada por apresentar baixa relação vermelho/vermelho-extremo (V/VE), e baixa densidade de fluxo de fóton fotossintético (PPFD) (Lee 1987). Esta variação na qualidade da luz pode causar efeitos significativos tanto na germinação de sementes e processos fotomor-

\footnotetext{
Departamento de Ciências Biológicas, Universidade de Franca. Av. Dr. Armando Salles Oliveira, 20I, CEP $14406-600$. Franca. SP. Brasil. e-mail: massa@rc.unesp.br

2. Departamento de Botânica, Instituto de Biocências, UNESP. C. Postal 199. CEP 13.506-900, Rio Claro. SP. Brasil. e-mail: zaia@francanet.com.br
} 
fogênicos de espécies vegetais florestais, como também influi na dinâmica de populações e sucessão ecológica (Bazzaz \& Pickett 1980; Bartley \& Frankland 1982; Garwood 1983; Withmore 1983; Smith 1986; Vazquez-Yanes \& Orosco-Segovia 1987). A percepção pelas plantas desta alteração no ambiente de luz é mediada pelo fitocromo, pigmento com duas formas fotoconversíveis ( Fv - inativa e Fve - ativa) que possuem absorção máxima nos comprimentos de onda de $660 \mathrm{~nm}$ (V) e 730nm (VE), respectivamente. Quando o fitocromo absorve a radiação vermelha, ocorre conversão de Fv em Fve e quando a absorção é de radiação VE, a forma Fve se converte para a forma Fv. Este equilíbrio entre as duas formas do fitocromo, denominado fotoequilíbrio do fitocromo, é diretamente proporcional à relação $\mathrm{V} / \mathrm{VE}$ existente na radiação, e que varia de 0,05 a 1,2 no ambiente natural (Holmes \& Smith 1977b).

Sob o dossel de uma floresta, as plantas experimentam gama variada de tipos de radiação. $\mathrm{O}$ ambiente de luz pode ser decomposto em dois componentes de radiação, direta e difusa. Radiação direta é aquela que chega ao solo diretamente do sol, enquanto radiação difusa é aquela proveniente de outras fontes (Chazdon \& Field 1987). Na literatura encontram-se muitos trabalhos que se referem à distribuição espectral da radiação em florestas, porém poucos levam em consideração a radiação difusa proveniente do céu nublado (Endler 1993). Entre outros dados, este autor encontrou que a relação V/VE sob o dossel é incrementada de quatro vezes em dias nublados quando comparada com dias de sol. Outros autores também fazem referência a este tipo de radiação (Federer \& Tanner 1966; Robertson 1966; Stoutjesdijk 1972; Holmes \& Smith 1977a).

Devido ao regime climático tipicamente chuvoso encontrado na Mata Atlântica de encosta, o céu se encontra encoberto durante grande parte do ano, predominando a radiação difusa. Esta radiação altera sensivelmente o regime de luz sob o dossel quando comparado com dias de sol. O objetivo deste trabalho foi comparar a relação $\mathrm{V} /$ VE e PPFD para os dois tipos de radiação que atuam sobre a Mata Atlântica, inferindo sobre a importância da radiação difusa na dinâmica populacional de espécies vegetais sob o dossel da floresta.

\section{Material e métodos}

Este trabalho foi conduzido em trecho de Mata Atlântica, pertencente ao Parque Estadual Intervales, Base de Saibadela $\left(24^{\circ} 14^{\prime}\right.$ 'S e $\left.48^{\circ} 04^{\prime} \mathrm{W}\right)$, SP, Brasil. Com altitude de 60-200m, caracteriza-se pelo relevo íngreme, pluviosidade média anual acima de $3.600 \mathrm{~mm}$ bem distribuídos durante o ano. A floresta é perenifolia, pouco perturbada, com dossel contínuo, variando entre 20-30m alt.

As medidas da distribuição espectral da radiação foram efetuadas nos meses de agosto de 1994, junho e agosto de 1995. As leituras de varredura do espectro de $300-1.100 \mathrm{~nm}$ em intervalos de $5 \mathrm{~nm}$ foram realizadas com espectroradiômetro LI-1.800 (Li-Cor Instruments) em 22 pontos sob o dossel da floresta e três pontos, em área aberta, sob condições de céu aberto (sol) e céu encoberto (nublado). Os mesmos pontos foram utilizados para ambas as condições (sol e nublado), e as leituras do espectro, apesar de terem sido realizadas em dias diferentes, ocorreram no mesmo horário. A partir do espectro de radiação foram obtidos os seguintes parâmetros: - PPFD, ou densidade de fluxo de fóton fotossintético $\left(\mathrm{mmol} . \mathrm{s}^{-1} \cdot \mathrm{m}^{-2}\right)$; relação V/VE, ou vermelho/vermelho-extremo, entre 655-665 e 725-735nm (Smith 1982). O fotoequilíbrio do fitocromo foi calculado segundo Mancinelli (1994). Os resultados foram comparados estatisticamente para os parâmetros PPFD e relação V/VE através dos testes "t pareado" e "Wilcoxon signed rank test" (Sokal \& Rohlf 1981).

\section{Resultados e discussão}

Fora do dossel da floresta, o espectro de radiação em condição de céu nublado não difere qualitativamente da radiação solar direta (Fig. 1). Com o céu nublado ocorre decréscimo da PPFD $(180,00 \pm 69,42)$ da ordem de aproximadamente $80 \%$, quando comparado aos valores encontrados de PPFD, sob condição de céu aberto (1147 \pm 213,43 ), no entanto a relação V/VE não se altera. 
Sob o dossel (Fig. 2), apenas a energia radiante sofre redução em dias nublados, devido, principalmente, aos comprimentos de onda acima de $700 \mathrm{~nm}$. Entretanto, a PPFD (400-700nm) não apresenta diferença quanto ao tipo de radiação. Os valores de PPFD encontrados para os dois tipos de radiação não apresentaram diferenças significativas entre si $(P=0,0597)$. Com relação aos valores de relação V/VE, para a radiação difusa, os valores encontrados foram significativamente maiores comparando-se com aqueles obtidos em dias de sol (Tab. 1). Como não ocorre alteração da relação V/VE fora do dossel em dias nublados, tais resultados sugerem que o incremento desta relação em baixo do dossel se deve, principalmente, ao decréscimo da radiação refletida e transmitida pelas folhas com comprimentos de onda acima de 700nm. De acordo com Lee (1987), as folhas transmitem e refletem pouca radiação entre os comprimentos de onda de 400-700nm, ocorrendo o oposto para os comprimentos de onda acima de $750 \mathrm{~nm}$.
Devido ao regime climático, a Mata Atlântica de encosta apresenta muitos dias de chuva ou encobertos durante o ano. Em 1995 foi realizado acompanhamento dos dias com céu aberto e céu encoberto, resultando em 169 dias sem sol (46\%), uma média de 14 dias de céu encoberto por mês. A luz proveniente da radiação difusa que chega até os estratos mais inferiores da floresta apresenta, em termos de PPFD, quantidades iguais àquelas apresentadas em dias de sol (Tab. 1). Além disso, em dias de céu encoberto, as plântulas experimentam acréscimo significativo na relação V/ VE (Tab. 1). Sob radiação solar direta, a relação de V/VE que chega até o solo sob o dossel da floresta variou de 0,14 a 0,38 . Estes valores são muito próximos aos obtidos por Lee (1987) em florestas tropicais. Sob radiação difusa, esta relação varia de 0,34 a 0,71 resultando em fotoequilíbrio do fitocromo da ordem de até 0,61 . Este incremento na relação V/VE pode ser fator de grande relevância para sementes fotoblásticas, que se aproveitariam deste regime intermediário

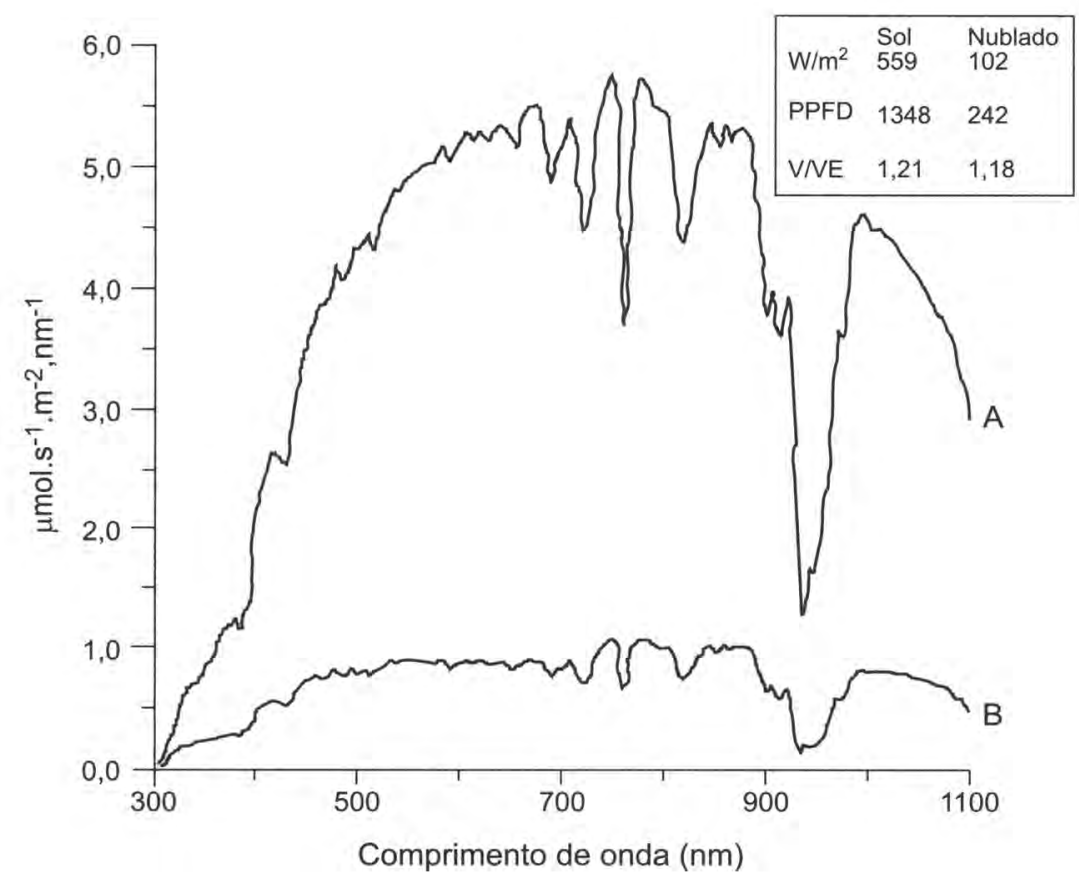

Figura 1. Distribuição espectral da radiação solar direta e difusa entre 300 e $1.100 \mathrm{~nm}$, numa área de clareira em condição de céu aberto (A) e nublado (B). Dados coletados em trecho de Mata Atlântica, no parque Estadual de Intervales, Base de Desenvolvimento do Saibadela, Sete Barras, SP. 


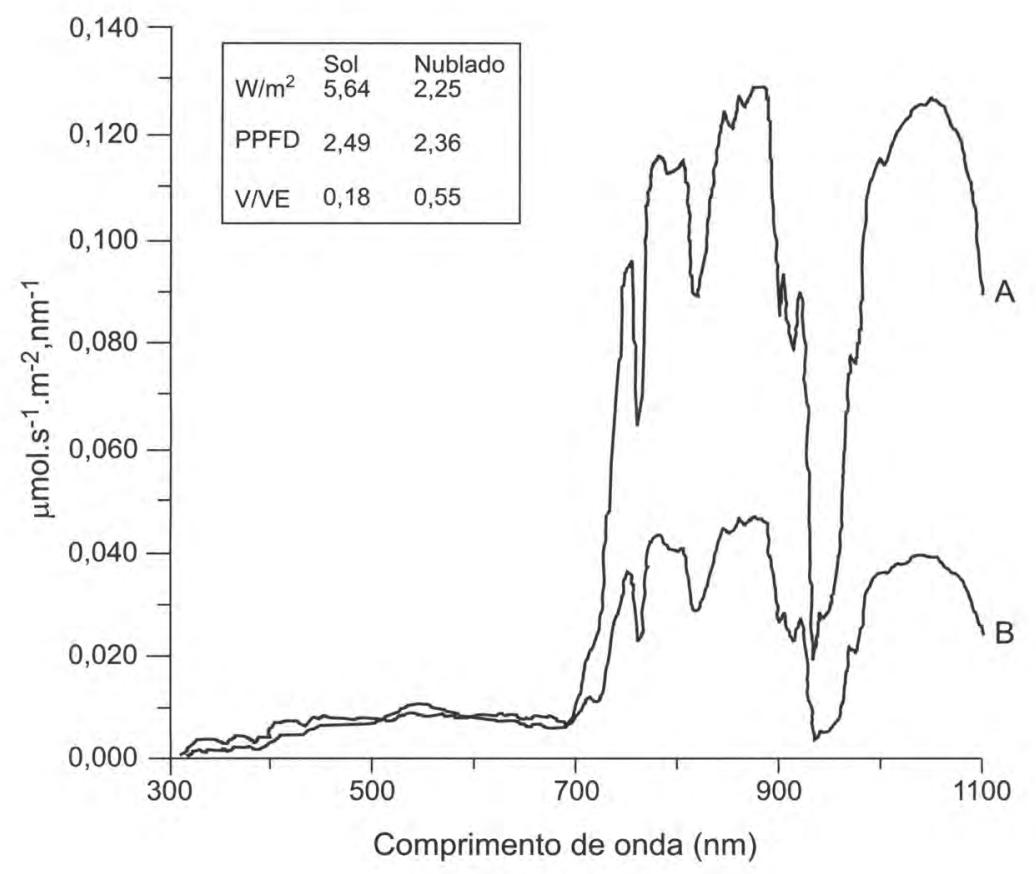

Figura 2. Distribuição espectral da radiação solar direta e difusa entre 300 e $1.100 \mathrm{~nm}$, numa área sob o dossel da floresta em condição de céu aberto (A) e nublado (B). Dados coletados em trecho de Mata Atlântica, no parque Estadual de Intervales, Base de Desenvolvimento do Saibadela, Sete Barras, SP.

de luz para promover sua germinação. Segundo Vazquez-Yanes (1980), não seria a intensidade, mas sim a qualidade da luz a responsável, em grande parte, pela germinação de espécies pioneiras constituintes do banco de sementes. Este autor realizou interessante experimento em condições naturais que consistia em colocar sementes de espécies pioneiras (Cecropia obtusifolia e Piper auritum) em placas de Petri cobertas por filtro vermelho, de modo que a relação V/VE fosse incrementada, sob o dossel de uma floresta úmida. As sementes nesta condição germinaram, ao contrário daquelas que não foram cobertas com o filtro.

Estudos com banco de sementes demonstram a necessidade de alta relação V/VE para que as sementes de espécies pioneiras que compõem este banco possam germinar, associando a este processo a abertura de clareiras na floresta (Denslow 1980; Augspurger 1984; Brokaw 1987). Segundo estes estudos, alta relação de V/VE ocorreria com a abertura de grandes clareiras, onde a radiação solar direta chegaria até o solo durante longo período do dia. Os resultados obtidos indicam que, embora os dados da literatura mostrem que no interior da floresta ocorra predominância de luz de comprimentos de onda acima de $700 \mathrm{~nm}$, isto é verdade somente para dias de sol. Porém, em dias nublados, esta relação varia consideravelmente, invalidando as generalizações sobre o comportamento das espécies vegetais no processo de sucessão secundária e, principalmente, em relação às espécies pioneiras.

Endler (1993) encontrou resultados que sugerem que o desenvolvimento de plântulas e outras espécies do sub-bosque pode ser incrementado enquanto o céu está nublado, sendo este desenvolvimento proporcional ao número de dias de céu encoberto. Sugere ainda que estas espécies podem se desenvolver mais rapidamente em florestas onde há predominância de céu encoberto do que em florestas com poucos dias nublados. Chazdon \& Fetcher (1984), por outro 
Tabela 1. Sumário dos valores obtidos durante dias de céu nublado e dias de sol para as áreas aberta e sob dossel de trecho de Mata Atlântica. no parque Estadual de Intervales. Base de Desenvolvimento do Saihadela. Sete Barras, SP.

\begin{tabular}{|c|c|c|c|c|c|}
\hline \multicolumn{2}{|c|}{ Tipo de local } & $\begin{array}{c}\text { Energia Radiante } \\
\qquad\left(\mathrm{W} \cdot \mathrm{m}^{-2}\right)\end{array}$ & $\begin{array}{c}\text { PPFD } \\
\left(\mu \mathrm{mol} . \mathrm{s}^{-1} \cdot \mathrm{m}^{-2}\right)\end{array}$ & V/VE & $\begin{array}{l}\text { Fotoequilíbrio } \\
\text { do Fitocromo }\end{array}$ \\
\hline \multicolumn{6}{|c|}{ Local Aberto } \\
\hline \multirow[t]{4}{*}{ Sol } & $n=3$ & & & & \\
\hline & média & 483,67 & 1147.00 & 1.21 & 0.698 \\
\hline & desvio-padrão & 76,01 & $21,3,43$ & 0,03 & 0,003 \\
\hline & $\min -\max$ & $407.00-559,00$ & $923.00-1348.00$ & $1,17-1,22$ & $0,695-0,701$ \\
\hline \multirow[t]{4}{*}{ Nublado } & $n=3$ & & & & \\
\hline & & $77,00 *$ & $180.00 \%$ & 1,22 & 0,700 \\
\hline & & 28.83 & 69.42 & 0,04 & 0,004 \\
\hline & & $46.00-103,00$ & $105.00-242.00$ & $1.18-1.25$ & $0.696-0.704$ \\
\hline \multicolumn{6}{|c|}{ Sob Dossel } \\
\hline \multirow[t]{4}{*}{ Sol } & $n=22$ & & & & \\
\hline & & 5,60 & 3.29 & 0.28 & 0,413 \\
\hline & & 2,84 & 1.34 & 0,07 & 0,055 \\
\hline & & $2.42-13,91$ & $1.79-6.32$ & $0,14-0,38$ & $0,280-0,490$ \\
\hline \multirow[t]{4}{*}{ Nublado } & $n=22$ & & & & \\
\hline & & $2,89 *$ & 3.10 & $0.538 *$ & $0.559 *$ \\
\hline & & 1.67 & 2.13 & 0,08 & 0,031 \\
\hline & & $0.63-7,12$ & $0.53-9.47$ & $0,34-0,71$ & $0,466-0,612$ \\
\hline
\end{tabular}

* Valores significativamente diferentes dos valores obtidos sob radiação solar direta ( $\mathrm{k}<0,05$ ), para um mesmo parâmetro e mesmo ambiente (aberto ou sob dossel).

lado, consideram que o desenvolvimento de espécies de sub-bosque estaria relacionado com "sunflecks". No entanto onde o dossel é denso o bastante, de modo que apenas em alguns poucos locais ocorram estes "sunflecks" poucas vezes ao dia, a radiação difusa proveniente do céu nublado pode resultar em diferença significativa. Em ambiente como a Mata Atlântica de encosta, onde o número de dias com céu encoberto é muito alto, resultando em aumento do valor da relação V/VE, período de sete dias nestas condições poderia desencadear o processo de germinação em algumas espécies pioneiras, como é o caso de Tibouchina pulchra (Zaia \& Takaki 1998). Sendo assim. a manutenção de fotoequilíbrio do fitocromo alto, sob o dossel, poderá levar a rápido esgotamento dessas espécies no banco de sementes, contrariando generalizações sobre o desenvolvimento e recrutamento de espécies vegetais relacionados apenas à radiação solar, proveniente de dias de sol dependentes da ocorrência de clareiras na vegetação.

\section{Agradecimentos}

Massanori Takaki, ao CNPq, pela concessão de Bolsa de Produtividade em Pesquisa; à FUNDUNESP, FAPESP e ao CNPq, pela concessão e auxílios; à Fundação Florestal pelo apoio logístico no Parque Estadual Intervales, Base Saibadela.

\section{Referências bibliográficas}

Augspurger. C. K. 1984. Seedling survival of tropical tree species: interactions of dispersal distance. light-gaps, and pathogens, Ecology 65: 1705-1712.

Bartley. M. R. \& Frankland. B. 1982. Analysis of the dual role of phytocrome in the photoinhibition of seed germination. Nature 300: 750-752.

Bazzaz. F. A.\& Pickett, S. T. A. 1980. Physiological ecology of tropical succession: A comparative review. Annual Review of Ecology and Systematics 11: 287-310.

Brokaw. N. V. L. 1987. Gap-phase regeneration of three pioneer tree species in a tropical forest. Journal of Ecology 75: 9-19. 
Chazdon. R. L. \& Fetcher, N. 1980. Photosynthetic light environments in a lowland tropical rain forest in Costa Rica. Journal of Ecology 72: 553-564

Chazdon, R. L.\& Field, C. B. 1987. Photographic estimation of photosynthetically active radiation: evaluation of a computerized technique. Oecologia 73: 525-532.

Denslow, J. S. 1980. Gap partitioning among tropical rainforest trees. Biotropica 12: 47-55.

Endler. J. A. 1993. The color of light in forests and its implications. Ecological Monographs 63: 1-23.

Federer. C. A. \& Tanner, C. B. 1966. Spectral distribution of light in the forest. Ecology 47: 555-560.

Garwood, N. C. 1983. Seed germination in a seazonal tropical forest in Panama: a community study. Ecological Monographs 53: 159-181.

Holmes. M. G. \& Smith, H. 1977a. The function of phytochrome in the natural environment. II. The influence of vegetation canopies on the spectral energy distribution of natural daylight. Photochemistry and Photobiology 25: $539-545$.

Holmes, M. G. \& Smith, H. 1977b. The function of phytochrome in the natural environment. IV. Light quality and plant development. Photochemistry and Photobiology 25: 551-557.

Lee, D. W. 1987. The spectral distribution of radiation in two neotropical rainforests. Biotropica 19: 161-166.

Mancinelli. A. L. 1994. The phisiology of phytochrome action Pp. 211-269. In: R.E. Kendrick \& G. H. M. Kronenberg (ed.) Photomorphogenesis in Plants. Kluwer Ac. Publ.. Dordrecht.

Robertson. G. W. 1966. The light composition of solar and sky spectra available to plants. Ecology 47: 640-643.

Smith. H. 1982. Light quality, photoperception, and plant estrategy. Annual Review of Plant Physiology 33: 481-518.

Smith. H. 1986. The perception of light quality. Pp 187-210. In R. E. Kendrick \& G. H. M. Kronenberg (ed). Photomorphogenesis in Plants. Martinus Nijhoff. Dordrecht.

Sokal. R. R. \& Rolph, F. J. 1981. Biometry. $2^{\text {nd }}$ Edition. W. H. Freeman and Co., San Francisco.

Stoutjesdijk. Ph. 1972. A note on the spectral transmition of light by tropical rainforest. Acta Botanica Neerlandica 21: $346-350$

Vazquez-Yanes. C. 1980. Light quality and seed germination in Cecropia obtusifolia and Piper auritum from a tropical rainforest in Mexico. Phyton 38: 33-35.

Vazquez-Yanes, C. \& Orozco-Segovia, A. 1987. Fisiologia ecologica de semillas en la estacion de biologia tropical "Los Tuxtlas". Veracruz, Mexico. Revista de Biologia Tropical 35: 85-96.

Whitmore. T. C. 1983. Secundary succession from seed in tropical rain forest. Forestry 44: 767-779.

Zaia, J. E. \& Takaki, M. 1998. Estudo da germinação de sementes de espécies arbóreas pioneiras: Tibouchina pulchra Cogn. e Tibouchina granulosa Cogn. (Melastomataceie). Acta Botanica Brasilica 12(3): 227-234. 